\title{
Response of Milling and Appearance Quality of Rice with Good Eating Quality to Temperature and Solar Radiation in Lower Reaches of Huai River
}

\author{
Nianbing Zhou $\mathbb{1}$, Haiyan Wei and Hongcheng Zhang * \\ Jiangsu Key Laboratory of Crop Genetics and Physiology/Jiangsu Co-Innovation Center for Modern Production \\ Technology of Grain Crops, Yangzhou University, Yangzhou 225009, China; iceazhou@163.com (N.Z.); \\ wei_haiyan@163.com (H.W.) \\ * Correspondence: hczhang@yzu.edu.cn
}

Citation: Zhou, N.; Wei, H.; Zhang, H. Response of Milling and Appearance Quality of Rice with Good Eating Quality to Temperature and Solar Radiation in Lower Reaches of Huai River. Agronomy 2021, 11, 77. https:/ / doi.org/10.3390/agronomy11010077

Received: 9 December 2020 Accepted: 29 December 2020 Published: 31 December 2020

Publisher's Note: MDPI stays neutral with regard to jurisdictional clai$\mathrm{ms}$ in published maps and institutional affiliations.

Copyright: (C) 2020 by the authors. Licensee MDPI, Basel, Switzerland. This article is an open access article distributed under the terms and conditions of the Creative Commons Attribution (CC BY) license (https:// creativecommons.org/licenses/by/ $4.0 /)$.

\begin{abstract}
The effects of temperature and solar radiation on milling and appearance quality of rice (Oryza sativa L.) were evaluated to find the optimal temperature and solar radiation for optimizing milling and appearance quality of rice in the lower reaches of Huai River. Field experiments were conducted with two medium-maturing japonica soft rice varieties (SMR), two late-maturing japonica soft rice varieties (SLR) and two late-maturing japonica non-soft rice varieties (LR) as experimental materials. Seeds were sown on 10 May (T1), 17 May (T2), 24 May (T3), 31 May (T4), 7 June (T5), 14 June (T6), and 21 June (T7) in 2017 and 2018. Compared with solar radiation, temperature was the main environmental factor affecting the milling and appearance quality of rice in the lower reaches of Huai River. Under the condition of ensuring relatively high-yield, the milling quality of SMR and SLR can reach the second grade of China's national standard of high quality paddy. The mean daily temperature $\left(\mathrm{T}_{\text {mean }}\right)$ range were $20.2-22.7^{\circ} \mathrm{C}$ and $20.4-22.0^{\circ} \mathrm{C}$ respectively. The temperature range for LR to obtain a relatively high-yield, good milling and appearance quality was $20.4-20.7^{\circ} \mathrm{C}$. The optimal sowing dates of SMR, SLR and LR were 15 May to 1 June, 15 May to 20 May and 15 May to 20 May, respectively.
\end{abstract}

Keywords: rice quality; temperature; solar radiation; sowing date

\section{Introduction}

The lower reaches of Huai River are located in the north of Jiangsu Province. This region is an important rice production area in Jiangsu Province. The total production and planting area of rice in the lower reaches of Huai River have increased since 1980, accounting for $42.1 \%$ and $43.5 \%$ of Jiangsu Province, respectively [1]. With a rapid development in the economy and improvement of living standards, the demand for high-quality rice has been increasing in China. The grain yield and quality are influenced by numerous factors such as varietal differences, agronomic practices, and climatic conditions [2-6]. Most studies have identified that the quality of field-grown rice strongly depends on the temperature and solar radiation throughout the grain filling period $[4,5]$, and ameliorating environmental conditions during the rice growing period by adjusting the sowing date is a practical and simple agronomic methods to improve the quality of rice [6,7].

Rice is primarily consumed as an intact kernel and the appearance quality reflects the ability to attract consumers. The milling and appearance quality are the foremost indicators for evaluating rice quality $[8,9]$. The milling appearance is an essential parameter of the final quality of rice, wheat, and other cereals final products, since that the milling process is able to generates the greatest and deepest changes in the final products [10-12], even higher than other essential processes such as kneading and baking [13,14]. Several studies have argued that high temperature at the heading-maturity stage of rice will shorten the filling time of rice, reduce the plumpness of grains and reduce the rate of brown rice, milled rice 
and head rice [15], In addition, a higher temperature will also cause the chalky grains and chalkiness degree to increase [16-18]. The low temperature during the grain filling stage will reduce the accumulation and transportation of assimilates, increase the "green rice rate", reduce the milling quality of rice [4,19], and increase the chalky grain and chalkiness degree $[20,21]$. The weak light environment at the filling stage of rice has also been reported to cause deterioration of rice milling and appearance quality [22,23]. Previous studies have suggested that the optimum temperature in the rice filling stage was $21.7-26.7^{\circ} \mathrm{C}$ [16]. Although there has been much research on the influence of temperatures or solar radiation on rice quality, the optimal range of temperatures or solar radiation for new rice varieties with good eating quality are not clear, and the adaptability of good eating quality rice in the lower reaches of Huai River is rarely reported. Therefore, it is necessary to study the response of milling and appearance quality to temperature and solar radiation in this region, and then the optimal sowing dates can be recommended for high-yielding and good quality production. Six rice varieties with good eating quality were selected as raw materials. Seven different temperature and solar radiation environments were established by setting different sowing dates in the lower reaches of Huai River. The objectives of the study were: (1) to reveal the rice requirement of temperature and solar radiation for high milling and appearance quality production in the lower reaches of Huai River; and (2) to propose an optimal range of sowing dates for high-yield, good milling and appearance quality production in this area.

\section{Materials and Methods}

\subsection{Plant Materials and Experimental Design}

The Field experiment was conducted during the rice cropping season in 2017 and repeated in 2018 in the same experimental field at Lingqiao township, Huai'an city, Jiangsu Province, China $\left(\mathrm{N} 33^{\circ} 35^{\prime}, \mathrm{E} 118^{\circ} 51^{\prime}\right)$. Huai' an city, which is located in the lower reaches of Huai River, it has a typical transitional monsoon climate in the north subtropical north warm temperate zone. The annual average temperature is about $14{ }^{\circ} \mathrm{C}$, the annual precipitation is about $960 \mathrm{~mm}$, the annual sunshine hours are about $2358.4 \mathrm{~h}$, and the frost free period is 239 days. The soil properties determined from the upper $20 \mathrm{~cm}$ layer were: organic matter $21.42 \mathrm{~g} \mathrm{~kg}^{-1}$, total $\mathrm{N} 1.59 \mathrm{~g} \mathrm{~kg}^{-1}$, available phosphorus $48.22 \mathrm{mg} \mathrm{kg}^{-1}$, and available potassium $98.28 \mathrm{mg} \mathrm{kg}^{-1}$.

The treatments were arranged in a split plot design with sowing dates as main plots and varieties as subplots, and the range of sowing dates in this study was designed to create contrasting environmental conditions that represent a wide range of situations for rice growth and development. Seven sowing dates were used, and six varieties were arranged in three replications within each sowing date. Planting dates were as follows: 10 May (T1), 17 May (T2), 24 May (T3), 31 May (T4), 7 June (T5), 14 June (T6), and 21 June (T7). Two medium-maturing japonica soft rice (SMR) varieties (Amylose content $<15 \%$ ) "Nangeng 2728" and "Nangeng 505", two late-maturing japonica soft rice (SLR) varieties (Amylose content $<15 \%$ ) "Nangeng 9108", "Fenggeng 1606", and two late-maturing japonica non-soft rice (LR) varieties (amylose content > 15\%) "Fenggeng 3227", "Wuyungeng 80" were used in 2017 and 2018. These six good eating quality varieties were chosen as they are currently the most widely cultivated in the lower reaches of Huai River. The varieties were raised in plastic plates and the seedlings were transplanted to the field 20 days after sowing at a hill spacing of $12 \mathrm{~cm} \times 30 \mathrm{~cm}$.

The total $\mathrm{N}$ application rate was $270 \mathrm{~kg} \mathrm{ha}^{-1}$. $\mathrm{N}$ was applied in three splits: $35 \%$ as basal fertilizer, $35 \%$ at tillering initiation, and $30 \%$ at panicle initiation. Nitrogen was applied as urea $(46.4 \% \mathrm{~N})$. For each plot, calcium superphosphate $\left(\mathrm{P}_{2} \mathrm{O}_{5}\right.$ content: $\left.12 \%\right)$ was applied as a basal fertilizer at the rate of $135 \mathrm{~kg} \mathrm{P}_{2} \mathrm{O}_{5} \mathrm{ha}^{-1}$. Similarly, potassium chloride $\left(\mathrm{K}_{2} \mathrm{O}\right.$ content: $\left.60 \%\right)$ was applied at a rate of $135 \mathrm{~kg} \mathrm{~K}_{2} \mathrm{O} \mathrm{ha}^{-1}$ as both basal fertilizer and at panicle initiation. The experimental field was flooded post-transplant and remained flooded until 7 days before maturity. Insects, diseases, and weeds were intensively controlled by chemicals to avoid losses in rice quality and yield. 


\subsection{Sample and Data Collection}

All rice plants were hand harvested. The final grain yield was adjusted to $14 \%$ moisture content. The China national standard of high-quality paddy (GB/T17891-2017) was an evaluation standard for rice quality promulgated by the National Food Administration Standard Quality Center, which has the general function of judging the quality of high quality paddy in China. According to the China national standard of high quality paddy, the grading index for milling quality and appearance of japonica rice is the head rice rate and chalkiness degree. The head rice rate should be equal or greater than $67 \%, 61 \%$ and $55 \%$, respectively, and the chalkiness degree should be equal or lesser than $2 \%, 4 \%$ and $6 \%$, respectively, when the milling and appearance quality reaches the first, second or third grade of China's national standard of high quality paddy. Rice quality analysis was performed according to the GB/T17891-2017 in this study. The brown rice, milled rice and head milled rice rate were expressed as percentages of the total grain weights, chalkiness was evaluated on 100 milled grains per plot. Chalkiness size was expressed as percentage of the total area of the kernel.

$$
\text { Chalkiness degree }(\%)=\text { Chalkiness rate } \times \text { Chalkiness size }
$$

The dates of heading and maturity were observed and recorded for each treatment. The daily air temperature and number of sunshine hours during the rice growing season in both experimental years were collected from a local weather observation point at the Huai'an Meteorological Station (Jiangsu Province, China).

\subsection{Calculation Methods and Statistical Analysis}

The effective accumulated temperature (EAT) in the determined growth duration expressed as ${ }^{\circ} \mathrm{C} d$ was calculated as:

$$
\mathrm{EAT}=\sum\left(\mathrm{T}-\mathrm{T}_{0}\right) \times \text { Growth duration, }
$$

where $\mathrm{T}$ and $\mathrm{T}_{0}\left(10^{\circ} \mathrm{C}\right.$ for japonica rice varieties) are the mean daily temperature and the biological zero temperature, respectively [19].

The environmental data for the period 2007-2016 in Huai'an City were collected from the National Meteorological Information Center of the China Meteorological Administration. The Angstrom-Prescott (AP) model was used to calculate daily global solar radiation from sunshine duration, because solar radiation could not be directly recorded at the meteorological station. It was calculated as follows:

$$
\frac{\mathrm{Q}}{\mathrm{Q}_{0}}=a+b \times \frac{\mathrm{S}}{\mathrm{S}_{0}}
$$

where $\mathrm{Q}\left(\mathrm{MJ} \mathrm{m} \mathrm{m}^{-2} \mathrm{~d}^{-1}\right)$ is global solar radiation, $\mathrm{Q}_{0}\left(\mathrm{MJ} \mathrm{m}^{-2}\right.$ day $\left.{ }^{-1}\right)$ is extraterrestrial solar radiation and total solar radiation of the ideal atmosphere, $S$ is the actual sunshine hours in a day, and $\mathrm{S}_{0}$ is the potential sunshine hours in a day. The constitute climatology coefficients $a$ and $b$ (Table 1), were described by Chen et al. as the extraterrestrial solar radiation and total solar radiation [24].

Table 1. The coefficients $a$ and $b$ for each month in the Angstrom-Prescott model.

\begin{tabular}{cccccccc}
\hline Coefficient & May & June & July & August & September & October & November \\
\hline$a$ & 0.211 & 0.239 & 0.303 & 0.272 & 0.304 & 0.290 & 0.206 \\
$b$ & 0.712 & 0.624 & 0.529 & 0.576 & 0.487 & 0.567 & 0.679 \\
\hline
\end{tabular}

The cumulative solar radiation (CSR) in the determined growth duration expressed as MJ $\mathrm{m}^{-2}$ was calculated as:

$$
\mathrm{CSR}=\sum \mathrm{Q} \times \text { Growth duration, }
$$


$\mathrm{Q}\left(\mathrm{MJ} \mathrm{m}^{-2} \mathrm{~d}^{-1}\right)$ is the daily global solar radiation

$$
\text { Relative grain yield }=\frac{\text { Yield }_{\mathrm{Ti}}}{\sum \text { Yield }_{\mathrm{Tn}}},
$$

Yield $_{\mathrm{Ti}}$ represents the yield of rice under Ti treatment, Yield $\mathrm{Tn}_{\mathrm{n}}$ represents the yield of the treatment that the rice can mature normally, SMR: $n=7$, SLR: $n=4$, LR: $n=4$.

Data were analyzed using analysis of variance (ANOVA) with SPSS 13.0. Means were compared by the least significant difference (LSD) test at the 0.05 probability level. In addition, the graphs were prepared with Microsoft Excel.

\section{Results}

\subsection{Effects of Sowing Date on Milling and Appearance Quality}

The six tested varieties experienced different temperature and solar radiation during their ripening phase due to a wide range in sowing dates. The late-maturing japonica soft rice(SLR) and late-maturing japonica non-soft rice(LR) cannot mature in T5, T6, and T7, and the harvest time (November 8) was taken as the deadline for rice growth, and it was used to calculate the effective accumulated temperature (EAT), mean daily temperature ( $\left.\mathrm{T}_{\text {mean }}\right)$, cumulative solar radiation (CSR), and mean daily solar radiation $\left(\mathrm{R}_{\text {mean }}\right)$. With the delay of sowing date, the EAT, $\mathrm{T}_{\text {mean }}, \mathrm{CSR}$, and $\mathrm{R}_{\text {mean }}$ of six rice varieties showed a decreasing trend at the stage from heading to maturity (Figures 1 and 2). The temperature under the same sowing date had similar values in the two year experiment, and the CSR, and $\mathrm{R}_{\text {mean }}$ in 2018 are slightly higher than those in 2017. The seven temperature and solar radiation treatments with significant differences were established for each rice variety by setting seven sowing dates in the same area.
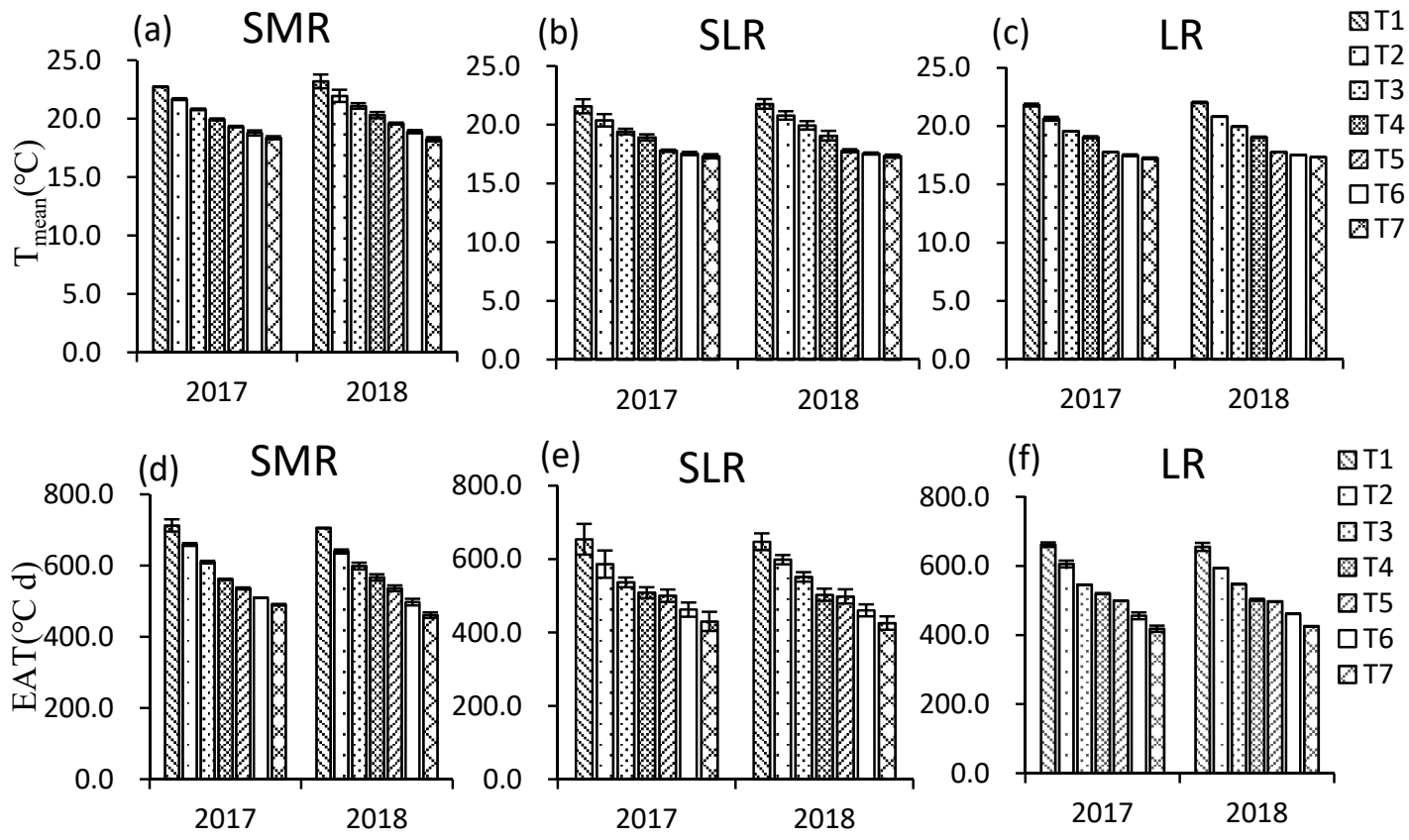

Figure 1. Differences in mean daily temperature $\left(\mathrm{T}_{\text {mean }},{ }^{\circ} \mathrm{C}\right)$ and effective accumulated temperature $\left(\mathrm{EAT},{ }^{\circ} \mathrm{C} \mathrm{d}\right)$ of rice at the stage from heading-maturity in the seven environmental condition treatments. $(\mathbf{a}-\mathbf{c})$ respesent the $\mathrm{T}_{\text {mean }}$ of SMR, SLR and LR at the stage from heading-maturity in the seven environmental condition treatments. (d-f) respesent the EAT of SMR, SLR and LR at the stage from heading-maturity in the seven environmental condition treatments. T1, T2, T3, T4, T5, T6, and T7 represent the sowing dates 10 May, 17 May, 24 May, 31 May, 7 June, 14 June, and 21 June. SMR: medium-maturing japonica soft rice, SLR: late-maturing japonica soft rice, LR: late-maturing japonica non-soft rice. 

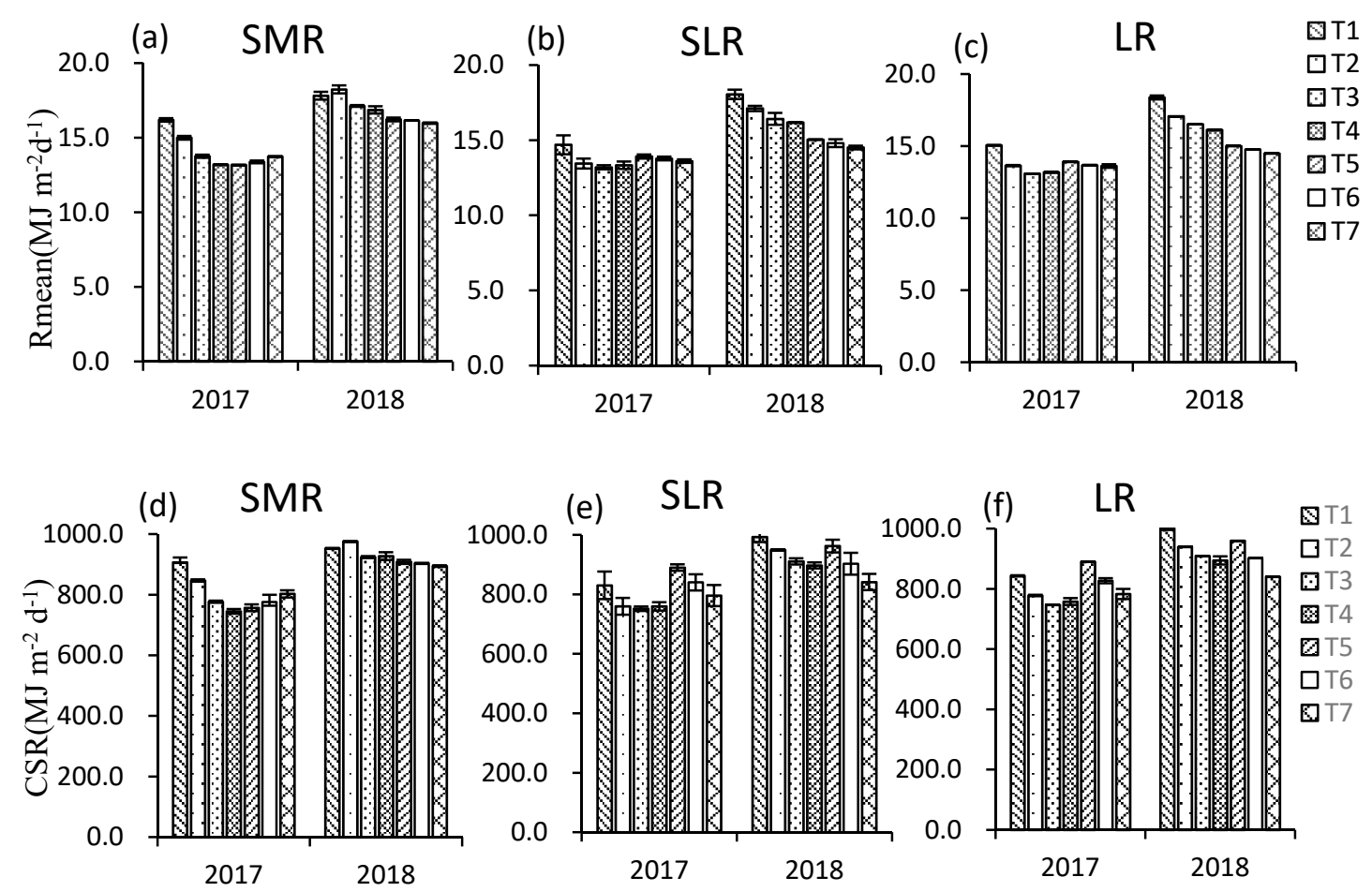

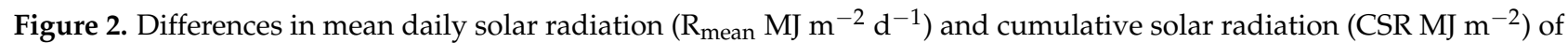
rice at the stage from heading-maturity in the seven environmental condition treatments. (a-c) respesent the $R_{\text {mean }}$ of $S M R$, SLR and LR at the stage from heading-maturity in the seven environmental condition treatments. (d-f) respesent the CSR of SMR, SLR and LR at the stage from heading-maturity in the seven environmental condition treatments.

In addition to the head milled rice rate, the milling quality had extremely significant differences among the years $(\mathrm{Y})$, types $(\mathrm{T})$, varieties $(\mathrm{V})$ and sowing dates $(\mathrm{S})$ (Table 2). The reason for this insignificant interaction of $Y \times S$ was due to the difference in milling quality in two years and the different changing trends of the six varieties under the conditions of the sowing date.

Table 2. Analysis of variance table for milling and appearance quality of rice among years, sowing dates and varieties.

\begin{tabular}{|c|c|c|c|c|c|c|}
\hline Analysis of Variance & $d f$ & Brown Rice Rate & Milled Rice Rate & Head Milled Rice Rate & Chalky Grains & Chalkiness Degree \\
\hline Year $(Y)$ & 1 & $50.956 * *$ & $11.653 * *$ & $3.004 \mathrm{NS}$ & $28.403^{* *}$ & $319.798 * *$ \\
\hline Type $(\mathrm{T})$ & 2 & $11.481 * *$ & $26.513 * *$ & $36.675 * *$ & $3488.960 * *$ & $55.969 * *$ \\
\hline Sowing date $(S)$ & 6 & $28.068 * *$ & $31.464 * *$ & $48.648^{* *}$ & $459.804^{* *}$ & $444.033^{* *}$ \\
\hline Variety (V) & 1 & $92.966^{* *}$ & $19.921 * *$ & $36.472 * *$ & $437.967 * *$ & $306.443 * *$ \\
\hline $\mathrm{Y} \times \mathrm{T}$ & 2 & $67.557 * *$ & $31.659 * *$ & $5.760 * *$ & $92.896^{* *}$ & $108.934 * *$ \\
\hline $\mathrm{Y} \times \mathrm{S}$ & 6 & $0.380 \mathrm{NS}$ & $1.105 \mathrm{NS}$ & $0.324 \mathrm{NS}$ & $29.270 * *$ & $2.974 *$ \\
\hline $\mathrm{Y} \times \mathrm{V}$ & 1 & $2.183 \mathrm{NS}$ & $35.045^{* *}$ & $1.692 \mathrm{NS}$ & $15.430 * *$ & $485.505^{* *}$ \\
\hline $\mathrm{T} \times \mathrm{S}$ & 12 & $58.571^{* *}$ & $76.606 * *$ & $47.115^{* *}$ & $141.089 * *$ & $59.200 * *$ \\
\hline $\mathrm{T} \times \mathrm{V}$ & 2 & $73.169 * *$ & $13.829 * *$ & $16.992 * *$ & $320.189 * *$ & 248.186 ** \\
\hline $\mathrm{S} \times \mathrm{V}$ & 6 & 2.276 * & $2.229 *$ & $0.613 \mathrm{NS}$ & $9.717^{* *}$ & $4.856^{* *}$ \\
\hline $\mathrm{Y} \times \mathrm{T} \times \mathrm{S}$ & 12 & $1.372 \mathrm{NS}$ & $2.633^{* *}$ & $0.581 \mathrm{NS}$ & $10.609 * *$ & $0.690 \mathrm{NS}$ \\
\hline $\mathrm{Y} \times \mathrm{T} \times \mathrm{V}$ & 2 & $3.730 *$ & $24.066 * *$ & $2.411 \mathrm{NS}$ & $11.225 * *$ & $119.044 * *$ \\
\hline $\mathrm{Y} \times \mathrm{S} \times \mathrm{V}$ & 6 & $1.328 \mathrm{NS}$ & $2.191 \mathrm{NS}$ & $0.206 \mathrm{NS}$ & $2.757 *$ & $1.477 \mathrm{NS}$ \\
\hline $\mathrm{T} \times \mathrm{S} \times \mathrm{V}$ & 12 & $3.660 * *$ & 2.264 * & $0.389 \mathrm{NS}$ & $10.793 * *$ & $5.388^{* *}$ \\
\hline $\mathrm{Y} \times \mathrm{T} \times \mathrm{S} \times \mathrm{V}$ & 12 & $0.627 \mathrm{NS}$ & $1.346 \mathrm{NS}$ & $0.291 \mathrm{NS}$ & $6.278 * *$ & $6.801 * *$ \\
\hline
\end{tabular}

** and * indicate significant difference at $p=0.01$ and $p=0.05$ levels, respectively, NS means not significant at the $p=0.05$ level.

A wide range in the milling quality of six tested varieties was observed across seven sowing dates. The milling quality of medium-maturing japonica soft rice (SMR) had been improved with the decrease in temperature and solar radiation. On the contrary, the SLR and LR showed a deterioration trend (Table 3 ). The brown rice rate, milled rice rate and head milled rice rate of SMR in T7 were $0.67-4.09 \%, 0.80-5.50 \%$ and $0.71-5.23 \%$ higher than 
those in $\mathrm{T} 1, \mathrm{~T} 2, \mathrm{~T} 3, \mathrm{~T} 4, \mathrm{~T} 5$ and $\mathrm{T} 6$, respectively. The brown rice rate, milled rice rate and head milled rice rate of SLR in T1 were $0.50-5.78 \%, 0.43-6.85 \%$ and $0.83-10.30 \%$ higher than those in T2, T3, T4, T5, T6 and T7, respectively. The T1 of LR were $0.29-3.36 \%, 0.42-6.86 \%$ and $0.72-8.93 \%$ higher than those in T2, T3, T4, T5, T6 and T7, respectively. Notably, a significant decrease was observed in the milling quality of SLR and LR in T5, T6 and T7.

Table 3. Differences in milling quality of rice under different temperature and solar radiation conditions.

\begin{tabular}{|c|c|c|c|c|c|c|c|}
\hline \multirow{2}{*}{ Variety } & \multirow{2}{*}{ Treatment } & \multicolumn{2}{|c|}{ Brown Rice Rate (\%) } & \multicolumn{2}{|c|}{ Milled Rice Rate (\%) } & \multicolumn{2}{|c|}{ Head Milled Rice Rate (\%) } \\
\hline & & 2017 & 2018 & 2017 & 2018 & 2017 & 2018 \\
\hline \multirow{7}{*}{ Nangeng2728 } & $\mathrm{T} 1$ & $83.17^{\mathrm{d}}$ & $82.45^{b}$ & $70.57^{\mathrm{d}}$ & $71.41^{b}$ & $63.54^{b}$ & $64.47^{c}$ \\
\hline & $\mathrm{T} 2$ & $83.59^{c, d}$ & $82.66^{b}$ & $71.40^{\mathrm{d}}$ & $71.34^{b}$ & $64.35^{b}$ & $64.84^{b, c}$ \\
\hline & T3 & $84.06^{c}$ & $83.02^{a, b}$ & $71.71^{\mathrm{c}, \mathrm{d}}$ & $71.66^{b}$ & $64.70^{a, b}$ & $65.25^{b, c}$ \\
\hline & $\mathrm{T} 4$ & $84.90^{b}$ & $83.43^{a, b}$ & $72.63^{b, c}$ & $72.53^{a}$ & $64.98^{\mathrm{a}, \mathrm{b}}$ & $66.09^{a, b, c}$ \\
\hline & $\mathrm{T} 5$ & $85.37^{b}$ & $83.53^{a, b}$ & $73.09^{a, b}$ & $72.66^{a}$ & $65.83^{a, b}$ & $66.33^{a, b}$ \\
\hline & T6 & $85.62^{b}$ & $83.67^{a, b}$ & $73.74^{\mathrm{a}, \mathrm{b}}$ & $72.92^{\mathrm{a}}$ & $66.60^{\mathrm{a}}$ & $66.63^{a, b}$ \\
\hline & $\mathrm{T} 7$ & $86.65^{a}$ & $84.22^{\mathrm{a}}$ & $74.25^{\mathrm{a}}$ & $73.24^{\mathrm{a}}$ & $67.29^{a}$ & $67.29^{\mathrm{a}}$ \\
\hline \multirow{7}{*}{ Nangeng505 } & $\mathrm{T} 1$ & $83.82^{c}$ & $82.06^{c}$ & $70.47^{d}$ & $70.10^{d}$ & $64.09^{c}$ & $64.88^{b}$ \\
\hline & $\mathrm{T} 2$ & $84.61^{b, c}$ & $82.12^{\mathrm{C}}$ & $70.66^{d}$ & $70.81^{\mathrm{c}, \mathrm{d}}$ & $64.17^{c}$ & $65.41^{\mathrm{a}, \mathrm{b}}$ \\
\hline & T3 & $84.61^{b, c}$ & $82.56^{c}$ & $71.63^{c, d}$ & $71.27^{\mathrm{c}, \mathrm{d}}$ & $64.78^{b, c}$ & $65.85^{\mathrm{a}, \mathrm{b}}$ \\
\hline & $\mathrm{T} 4$ & $85.72^{a, b}$ & $83.18^{b, c}$ & $72.69^{b, c}$ & $71.77^{b, c}$ & $65.75^{a, b, c}$ & $66.30^{a, b}$ \\
\hline & $\mathrm{T} 5$ & $86.10^{a, b}$ & $83.51^{a, b, c}$ & $73.25^{b, c}$ & $72.19^{a, b, c}$ & $65.81^{a, b, c}$ & $66.74^{\mathrm{a}, \mathrm{b}}$ \\
\hline & T6 & $86.29^{a}$ & $84.07^{\mathrm{a}, \mathrm{b}}$ & $72.97^{a, b}$ & $72.88^{a, b}$ & $66.52^{a, b}$ & $67.16^{\mathrm{a}}$ \\
\hline & $\mathrm{T} 7$ & $87.17^{\mathrm{a}}$ & $84.66^{\mathrm{a}}$ & $74.53^{\mathrm{a}}$ & $73.72^{\mathrm{a}}$ & $67.03^{a}$ & $67.45^{\mathrm{a}}$ \\
\hline \multirow{7}{*}{ Nangeng9108 } & $\mathrm{T} 1$ & $85.31^{a}$ & $85.25^{a}$ & $73.77^{a}$ & $73.65^{a}$ & $67.63^{a}$ & $67.68^{a}$ \\
\hline & $\mathrm{T} 2$ & $84.89^{a}$ & $85.09^{a}$ & $73.20^{\mathrm{a}}$ & $73.25^{\mathrm{a}}$ & $67.23^{a}$ & $67.25^{\mathrm{a}}$ \\
\hline & T3 & $84.76^{\mathrm{a}}$ & $84.84^{\mathrm{a}}$ & $73.12^{\mathrm{a}}$ & $73.18^{a}$ & $66.58^{a}$ & $66.78^{a}$ \\
\hline & $\mathrm{T} 4$ & $83.75^{a}$ & $83.70^{b}$ & $73.05^{a}$ & $73.07^{a}$ & $66.17^{a}$ & $66.23^{a}$ \\
\hline & $\mathrm{T} 5$ & $81.08^{b}$ & $81.83^{c}$ & $71.36^{b}$ & $71.62^{b}$ & $64.37^{\mathrm{b}}$ & $63.67^{\mathrm{b}}$ \\
\hline & T6 & $80.35^{b}$ & $81.35^{c}$ & $70.43^{b}$ & $70.69^{b}$ & $63.25^{b, c}$ & $62.77^{b, c}$ \\
\hline & $\mathrm{T} 7$ & $80.00^{b}$ & $79.01^{\mathrm{d}}$ & $68.53^{c}$ & $68.60^{c}$ & $62.36^{c}$ & $61.61^{\mathrm{c}}$ \\
\hline \multirow{7}{*}{ Fenggeng1606 } & $\mathrm{T} 1$ & $86.72^{a}$ & $86.83^{a}$ & $73.87^{a}$ & $73.57^{\mathrm{a}}$ & $67.81^{a}$ & $66.58^{a}$ \\
\hline & $\mathrm{T} 2$ & $86.29^{a, b}$ & $86.14^{\mathrm{a}, \mathrm{b}}$ & $73.59^{a}$ & $73.34^{\mathrm{a}}$ & $66.88^{a, b}$ & $65.92^{\mathrm{a}}$ \\
\hline & T3 & $85.22^{a, b, c}$ & $86.58^{\mathrm{a}, \mathrm{b}}$ & $73.25^{a}$ & $72.97^{\mathrm{a}}$ & $65.90^{\mathrm{a}, \mathrm{b}}$ & $65.53^{a}$ \\
\hline & $\mathrm{T} 4$ & $84.99^{b, c}$ & $85.57^{\mathrm{b}}$ & $72.25^{b}$ & $72.49^{a, b}$ & $64.57^{b, c}$ & $64.71^{\mathrm{a}}$ \\
\hline & $\mathrm{T} 5$ & $84.61^{c, d}$ & $84.27^{c}$ & $71.66^{b}$ & $71.45^{b, c}$ & $62.50^{c, d}$ & $61.69^{b}$ \\
\hline & T6 & $83.32^{d}$ & $84.03^{c}$ & $70.30^{c}$ & $71.07^{c, d}$ & $61.03^{\mathrm{d}}$ & $61.28^{b}$ \\
\hline & $\mathrm{T} 7$ & $83.16^{\mathrm{d}}$ & $83.66^{c}$ & $69.63^{c}$ & $69.87^{\mathrm{d}}$ & $60.43^{\mathrm{d}}$ & $60.20^{b}$ \\
\hline \multirow{7}{*}{ Fenggeng3227 } & $\mathrm{T} 1$ & $84.85^{a}$ & $84.63^{a}$ & $75.86^{a}$ & $75.64^{a}$ & $67.49^{a}$ & $67.54^{\mathrm{a}}$ \\
\hline & $\mathrm{T} 2$ & $84.68^{a, b}$ & $84.27^{\mathrm{a}, \mathrm{b}}$ & $72.06^{b}$ & $75.32^{\mathrm{a}}$ & $66.94^{\mathrm{a}}$ & $67.15^{\mathrm{a}}$ \\
\hline & $\mathrm{T} 3$ & $84.14^{\mathrm{a}, \mathrm{b}, \mathrm{c}}$ & $83.98^{a, b, c}$ & $71.66^{b, c}$ & $74.98^{\mathrm{a}}$ & $66.23^{a, b}$ & $66.87^{\mathrm{a}}$ \\
\hline & $\mathrm{T} 4$ & $83.97^{a, b, c}$ & $83.57^{a, b, c}$ & $71.06^{b, c}$ & $74.47^{\mathrm{a}, \mathrm{b}}$ & $65.20^{\mathrm{b}}$ & $66.19^{a, b}$ \\
\hline & $\mathrm{T} 5$ & $83.19^{a, b, c}$ & $83.77^{a, b, c}$ & $70.89^{b, c}$ & $73.57^{b, c}$ & $63.49^{c}$ & $64.50^{b, c}$ \\
\hline & T6 & $82.88^{b, c}$ & $83.20^{b, c}$ & $71.64^{b, c}$ & $73.17^{b, c}$ & $62.31^{\mathrm{c}, \mathrm{d}}$ & $64.06^{c}$ \\
\hline & $\mathrm{T} 7$ & $82.68^{c}$ & $82.80^{c}$ & $72.65^{c}$ & $72.96^{c}$ & $61.82^{\mathrm{d}}$ & $62.99^{c}$ \\
\hline \multirow{7}{*}{ Wuyungeng80 } & $\mathrm{T} 1$ & $85.40^{a}$ & $84.82^{a}$ & $74.48^{a}$ & $73.61^{a}$ & $66.84^{\mathrm{a}}$ & $66.66^{a}$ \\
\hline & $\mathrm{T} 2$ & $84.94^{\mathrm{a}}$ & $84.69^{a}$ & $73.86^{a, b}$ & $73.30^{\mathrm{a}}$ & $66.43^{a}$ & $65.47^{a, b}$ \\
\hline & $\mathrm{T} 3$ & $84.50^{a, b}$ & $83.81^{b}$ & $72.98^{b, c}$ & $72.85^{\mathrm{a}, \mathrm{b}}$ & $65.04^{b}$ & $64.78^{b}$ \\
\hline & $\mathrm{T} 4$ & $83.46^{b, c}$ & $83.53^{b}$ & $72.50^{c}$ & $72.13^{a, b, c}$ & $64.65^{b}$ & $63.92^{b, c}$ \\
\hline & $\mathrm{T} 5$ & $83.13^{b, c}$ & $82.91^{c}$ & $71.90^{c}$ & $71.58^{b, c}$ & $62.67^{c}$ & $62.81^{\mathrm{c}, \mathrm{d}}$ \\
\hline & T6 & $82.79^{c}$ & $82.43^{c, d}$ & $70.52^{d}$ & $71.04^{c}$ & $61.72^{\mathrm{c}, \mathrm{d}}$ & $62.61^{\mathrm{c}, \mathrm{d}}$ \\
\hline & $\mathrm{T} 7$ & $82.03^{c}$ & $82.71^{\mathrm{d}}$ & $70.05^{d}$ & $70.95^{c}$ & $61.50^{\mathrm{d}}$ & $61.31^{\mathrm{d}}$ \\
\hline
\end{tabular}

Values followed by different lowercase letters within a column are significantly different at the $p=0.05$ level. T1, T2, T3, T4, T5, T6, and T7 represent the sowing dates 10 May, 17 May, 24 May, 31 May, 7 June, 14 June, and 21 June.

The milling quality of the same type of rice varieties was similar on the same sowing date. The milling quality of SLR and LR was better than that of SMR in T1, T2 and T3. While in T5, T6 and T7, the milling quality of SMR was better than that of SLR and LR.

The chalky grain rate and chalkiness degree of SMR decreased with the reduction in temperature and solar radiation. The chalky grain and chalkiness degree of T1 were $0.37-146.23 \%$ and $1.97-187.67 \%$ higher than those in other treatments, respectively (Table 4). 
The chalky grain and chalkiness degree of SLR and LR decreased first and then increased with the reduction of temperature and solar radiation. The significant increase in chalky grain and chalkiness degree of SLR and LR were related to the incomplete maturity in T5, T6 and T7.

Table 4. Differences in appearance quality of rice under different temperature and solar radiation conditions.

\begin{tabular}{|c|c|c|c|c|c|}
\hline \multirow{2}{*}{ Variety } & \multirow{2}{*}{ Treatment } & \multicolumn{2}{|c|}{ Chalky Grains (\%) } & \multicolumn{2}{|c|}{ Chalkiness Degree (\%) } \\
\hline & & 2017 & 2018 & 2017 & 2018 \\
\hline \multirow{7}{*}{ Nangeng2728 } & $\mathrm{T} 1$ & $53.61^{a}$ & $53.89^{a}$ & $7.00^{a}$ & $6.68^{a}$ \\
\hline & $\mathrm{T} 2$ & $53.31^{\mathrm{a}}$ & $53.69^{a}$ & $6.86^{b}$ & $6.36^{a, b}$ \\
\hline & T3 & $53.07^{\mathrm{a}}$ & $48.11^{b}$ & $6.27^{c}$ & $6.03^{b}$ \\
\hline & $\mathrm{T} 4$ & $38.26^{b}$ & $44.89^{c}$ & $5.76^{\mathrm{d}}$ & $5.52^{\mathrm{c}}$ \\
\hline & $\mathrm{T} 5$ & $30.09^{c}$ & $44.42^{c}$ & $5.46^{\mathrm{e}}$ & $5.24^{\mathrm{c}}$ \\
\hline & $\mathrm{T} 6$ & $26.63^{d}$ & $38.00^{d}$ & $5.32^{f}$ & $4.72^{\mathrm{d}}$ \\
\hline & $\mathrm{T} 7$ & $21.77^{\mathrm{e}}$ & $29.20^{\mathrm{e}}$ & $5.18^{\mathrm{g}}$ & $4.46^{\mathrm{d}}$ \\
\hline \multirow{7}{*}{ Nangeng505 } & $\mathrm{T} 1$ & $52.08^{a}$ & $52.44^{\mathrm{a}}$ & $6.36^{\mathrm{a}}$ & $8.56^{\mathrm{a}}$ \\
\hline & $\mathrm{T} 2$ & $50.11^{\mathrm{a}, \mathrm{b}}$ & $49.80^{\mathrm{a}, \mathrm{b}}$ & $5.69^{b}$ & $7.73^{a, b}$ \\
\hline & $\mathrm{T} 3$ & $47.39^{b}$ & $47.49^{b}$ & $4.65^{c}$ & $7.22^{b, c}$ \\
\hline & $\mathrm{T} 4$ & $40.07^{\mathrm{c}}$ & $41.43^{c}$ & $3.24^{\mathrm{d}}$ & $6.44^{c, d}$ \\
\hline & $\mathrm{T} 5$ & $35.96^{\mathrm{c}, \mathrm{d}}$ & $37.96^{\mathrm{c}, \mathrm{d}}$ & $3.09^{\mathrm{d}}$ & $6.14^{\mathrm{d}, \mathrm{e}}$ \\
\hline & $\mathrm{T} 6$ & $32.54^{\mathrm{d}}$ & $35.73^{d}$ & $2.86^{\mathrm{e}}$ & $5.67^{\mathrm{d}, \mathrm{e}}$ \\
\hline & $\mathrm{T} 7$ & $28.23^{e}$ & $26.18^{\mathrm{e}}$ & $2.21^{\mathrm{f}}$ & $5.30^{\mathrm{e}}$ \\
\hline \multirow{7}{*}{ Nangeng9108 } & $\mathrm{T} 1$ & $46.58^{a}$ & $46.70^{a}$ & $6.46^{\mathrm{a}}$ & $7.84^{\mathrm{a}}$ \\
\hline & $\mathrm{T} 2$ & $44.95^{\mathrm{a}}$ & $39.04^{b}$ & $5.70^{\mathrm{b}}$ & $6.34^{b}$ \\
\hline & $\mathrm{T} 3$ & $30.41^{c}$ & $32.15^{c}$ & $4.40^{\mathrm{d}}$ & $4.87^{\mathrm{c}}$ \\
\hline & $\mathrm{T} 4$ & $23.39^{d}$ & $21.02^{\mathrm{e}}$ & $3.54^{\mathrm{e}}$ & $3.89^{c}$ \\
\hline & $\mathrm{T} 5$ & $28.83^{c}$ & $27.81^{\mathrm{d}}$ & $4.06^{\mathrm{d}}$ & $4.44^{\mathrm{d}}$ \\
\hline & $\mathrm{T} 6$ & $40.24^{b}$ & $33.71^{c}$ & $5.01^{\mathrm{c}}$ & $5.61^{\mathrm{e}}$ \\
\hline & $\mathrm{T} 7$ & $42.45^{\mathrm{a}, \mathrm{b}}$ & $37.97^{b}$ & $5.41^{b, c}$ & $5.88^{f}$ \\
\hline \multirow{7}{*}{ Fenggeng1606 } & $\mathrm{T} 1$ & $36.95^{a}$ & $30.05^{a}$ & $7.43^{a}$ & $7.82^{a}$ \\
\hline & $\mathrm{T} 2$ & $30.16^{b}$ & $23.90^{b}$ & $6.54^{\mathrm{b}}$ & $7.14^{\mathrm{b}}$ \\
\hline & T3 & $20.87^{c, d}$ & $19.93^{c}$ & $4.56^{\mathrm{d}}$ & $6.06^{\mathrm{d}, \mathrm{e}}$ \\
\hline & $\mathrm{T} 4$ & $18.38^{\mathrm{d}}$ & $19.58^{c}$ & $3.55^{\mathrm{e}}$ & $5.51^{\mathrm{f}}$ \\
\hline & $\mathrm{T} 5$ & $20.71^{\mathrm{c}, \mathrm{d}}$ & $19.84^{\mathrm{C}}$ & $4.25^{\mathrm{d}}$ & $5.82^{e, f}$ \\
\hline & $\mathrm{T} 6$ & $21.80^{c, d}$ & $20.99 b, c$ & $5.52^{c}$ & $6.55^{\mathrm{s}, \mathrm{d}}$ \\
\hline & $\mathrm{T} 7$ & $24.97^{c}$ & $21.62^{b, c}$ & $6.21^{b}$ & $7.02^{b, c}$ \\
\hline \multirow{7}{*}{ Fenggeng 3227} & $\mathrm{~T} 1$ & $33.89^{a}$ & $23.33^{a}$ & $6.99^{a}$ & $5.29^{a}$ \\
\hline & $\mathrm{T} 2$ & $28.68^{b}$ & $20.92^{\mathrm{a}, \mathrm{b}}$ & $5.60^{b}$ & $4.89^{\mathrm{a}, \mathrm{b}}$ \\
\hline & T3 & $17.32^{d}$ & $18.07^{c, d}$ & $3.94^{\mathrm{d}}$ & $3.60^{c, d}$ \\
\hline & $\mathrm{T} 4$ & $14.51^{\mathrm{d}}$ & $15.53^{\mathrm{d}}$ & $3.18^{\mathrm{e}}$ & $2.87^{\mathrm{e}}$ \\
\hline & $\mathrm{T} 5$ & $14.91^{\mathrm{d}}$ & $17.36^{\mathrm{c}, \mathrm{d}}$ & $3.55^{\mathrm{d}, \mathrm{e}}$ & $3.37^{\mathrm{d}, \mathrm{e}}$ \\
\hline & $\mathrm{T} 6$ & $22.05^{c}$ & $18.71^{b, c}$ & $4.53^{c}$ & $3.99 \mathrm{~d}, \mathrm{e}, \mathrm{c}$ \\
\hline & $\mathrm{T} 7$ & $24.61^{c}$ & $18.85^{b, c}$ & $5.47^{\mathrm{c}}$ & $4.68^{b}$ \\
\hline \multirow{7}{*}{ Wuyungeng80 } & $\mathrm{T} 1$ & $33.88^{a}$ & $22.96^{\mathrm{a}}$ & $7.32^{\mathrm{a}}$ & $8.37^{a}$ \\
\hline & $\mathrm{T} 2$ & $27.50^{b}$ & $19.63^{b}$ & $6.80^{b}$ & $7.20^{b}$ \\
\hline & T3 & $16.11^{\mathrm{d}}$ & $16.44^{\mathrm{C}}$ & $5.20^{\mathrm{e}}$ & $5.40^{\mathrm{e}}$ \\
\hline & $\mathrm{T} 4$ & $14.27^{\mathrm{d}}$ & $16.21^{\mathrm{c}}$ & $4.11^{\mathrm{g}}$ & $4.24^{\mathrm{f}}$ \\
\hline & $\mathrm{T} 5$ & $14.78^{\mathrm{d}}$ & $16.38^{c}$ & $4.79^{\mathrm{f}}$ & $5.21^{\mathrm{e}}$ \\
\hline & $\mathrm{T} 6$ & $21.90^{c}$ & $17.38^{b, c}$ & $5.65^{\mathrm{d}}$ & $6.19^{d}$ \\
\hline & $\mathrm{T} 7$ & $26.44^{b}$ & $18.66^{b, c}$ & $6.26^{c}$ & $6.83^{c}$ \\
\hline
\end{tabular}

Values followed by different lowercase letters within a column are significantly different at the $p=0.05$ level.

For different types of varieties, the LR had the best appearance quality under the same sowing date. 


\subsection{Correlation between Rice Milling Quality, Appearance Quality and Temperature or Solar Radiation}

The milling quality of the three types of rice showed a significantly correlation with $\mathrm{T}_{\text {mean }}$ or EAT at the stage from heading to maturity. The correlation coefficients of milling quality with $R_{\text {mean }}$ or CSR were smaller than the correlation coefficients of milling quality with $\mathrm{T}_{\text {mean }}$ or EAT (Table 5). The $\mathrm{T}_{\text {mean }}$ and, EAT at the stage from heading to maturity showed a positive correlation with chalky grain and chalkiness degree. However, marked differences were observed in correlations between solar radiation and appearance quality in two years. These results indicated that the influence of temperature on rice milling and appearance quality was greater than that of solar radiation.

Table 5. Correlation analysis between rice quality and environmental factors at the stage from heading to maturity.

\begin{tabular}{|c|c|c|c|c|c|c|c|c|c|}
\hline \multirow{2}{*}{ Type } & \multirow{2}{*}{ Rice Quality } & \multicolumn{2}{|c|}{$\mathrm{T}_{\text {mean }}$} & \multicolumn{2}{|c|}{ EAT } & \multicolumn{2}{|c|}{$\mathbf{R}_{\text {mean }}$} & \multicolumn{2}{|c|}{ CSR } \\
\hline & & 2017 & 2018 & 2017 & 2018 & 2017 & 2018 & 2017 & 2018 \\
\hline \multirow{5}{*}{ SMR } & brown rice rate $(\%)$ & $-0.939^{* *}$ & $-0.933^{* *}$ & $-0.909^{* *}$ & $-0.938^{* *}$ & $-0.716^{* *}$ & $-0.867^{* *}$ & $-0.538 *$ & $-0.844^{* *}$ \\
\hline & milled rice rate (\%) & $-0.963^{* *}$ & $-0.904^{* *}$ & $-0.967^{* *}$ & $-0.912^{* *}$ & $-0.765^{* *}$ & $-0.824^{* *}$ & $-0.654 *$ & $-0.803^{* *}$ \\
\hline & head milled rice $(\%)$ & $-0.962 * *$ & $-0.981 * *$ & $-0.948^{* *}$ & $-0.974^{* *}$ & $-0.717^{* *}$ & $-0.921^{* *}$ & $-0.609 *$ & $0.853^{* *}$ \\
\hline & Chalky grains (\%) & $0.924^{* *}$ & $0.943^{* *}$ & $0.921^{* *}$ & $0.945^{* *}$ & $0.690 * *$ & $0.881^{* *}$ & $0.568 *$ & $0.846^{* *}$ \\
\hline & Chalkiness degree (\%) & $0.737^{* *}$ & $0.793 * *$ & $0.692 * *$ & $0.803^{* *}$ & $0.617 *$ & $0.716^{* *}$ & 0.440 & $0.711^{* *}$ \\
\hline \multirow{5}{*}{ SLR } & brown rice rate $(\%)$ & $0.719^{* *}$ & $0.683^{* *}$ & $0.621^{*}$ & $0.628 *$ & 0.045 & $0.686^{* *}$ & 0.045 & $0.686^{* *}$ \\
\hline & milled rice rate $(\%)$ & $0.862^{* *}$ & $0.864^{* *}$ & $0.837^{* *}$ & $0.860^{* *}$ & 0.083 & $0.875^{* *}$ & 0.083 & $0.875^{* *}$ \\
\hline & head milled rice $(\%)$ & $0.917^{* *}$ & $0.931 * *$ & $0.905^{* *}$ & $0.909^{* *}$ & 0.130 & $0.931^{* *}$ & 0.130 & $0.931^{* *}$ \\
\hline & Chalky grains (\%) & 0.475 & 0.456 & 0.531 & 0.494 & 0.537 * & 0.440 & $0.537^{*}$ & 0.440 \\
\hline & Chalkiness degree $(\%)$ & 0.440 & 0.413 & 0.387 & 0.388 & 0.451 & 0.396 & 0.451 & 0.396 \\
\hline \multirow{5}{*}{ LR } & brown rice rate $(\%)$ & $0.962^{* *}$ & $0.927^{* *}$ & $0.964^{* *}$ & $0.932 * *$ & 0.369 & $0.916^{* *}$ & -0.137 & $0.706^{* *}$ \\
\hline & milled rice rate (\%) & $0.881^{* *}$ & $0.675^{* *}$ & $0.895^{* *}$ & $0.642 *$ & 0.634 * & $0.673^{* *}$ & 0.127 & 0.423 \\
\hline & head milled rice $(\%)$ & $0.956^{* *}$ & $0.859 * *$ & $0.945^{* *}$ & $0.839^{* *}$ & 0.300 & $0.859^{* *}$ & -0.221 & $0.601 *$ \\
\hline & Chalky grains (\%) & $0.553 *$ & $0.664^{* *}$ & 0.489 & $0.677^{* *}$ & $0.730 * *$ & $0.648 *$ & 0.114 & 0.532 \\
\hline & Chalkiness degree $(\%)$ & 0.496 & 0.352 & 0.442 & 0.369 & $0.667^{* *}$ & 0.313 & 0.119 & 0.261 \\
\hline
\end{tabular}

** and * respectively represent extremely significant correlation and significant correlation. $\mathrm{r}_{0.01}=0.661 ; \mathrm{r}_{0.05}=0.533$.

Under conditions of complete maturity, the head milled rice rate and chalkiness degree showed a significant correlation with EAT or $\mathrm{T}_{\text {mean }}$ (Figures 3-6). The result showed that, to obtain the second grade of milling and appearance quality of China's national standard GB/T 17891-2017, the demand of temperature at the stage from heading to maturity for SMR were lower than those of SLR and LR (Tables 6 and 7).
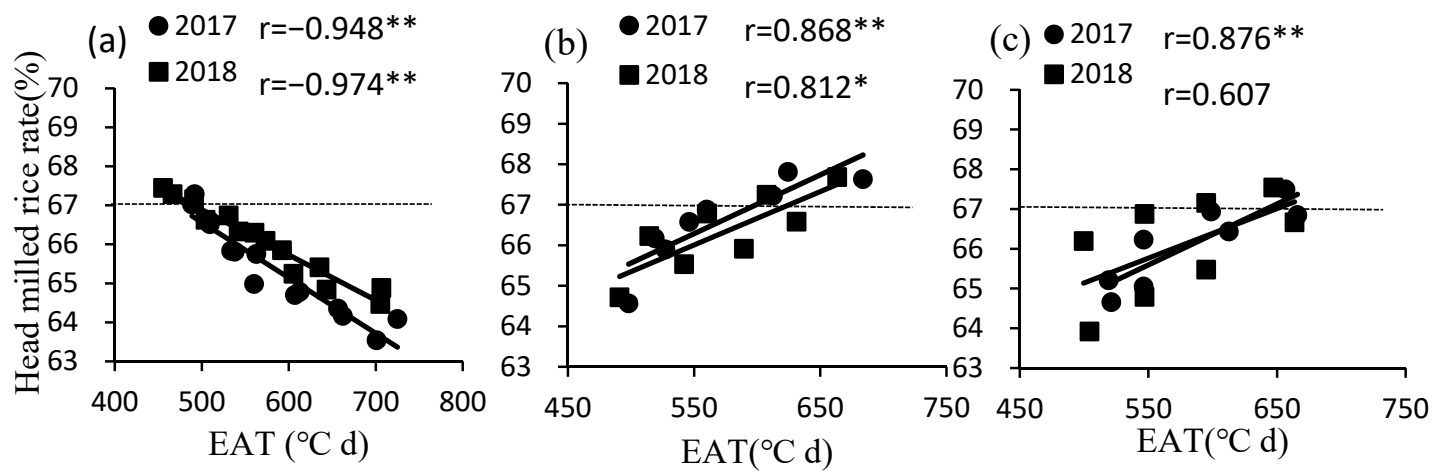

Figure 3. The correlation between head milled rice rate and the EAT of rice at stage of heading-maturity (a): SMR, $n=14$, (b): SLR, $n=8$, (c): LR, $n=8$, the (immature treatment including T5, T6 and T7 was removed from SLR and LR). * and ** indicate $p<0.05$ and $p<0.01$, respectively. 


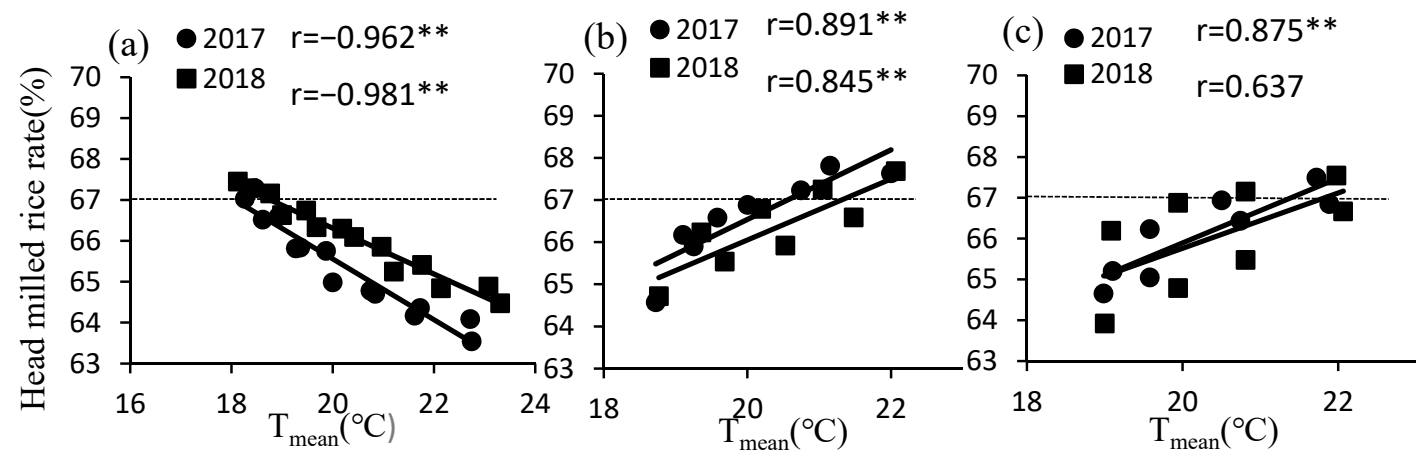

Figure 4. The correlation between head milled rice rate and the $\mathrm{T}_{\text {mean }}$ of rice at stage of heading-maturity (a): $\mathrm{SMR}$, $n=14,(\mathbf{b})$ : SLR, $n=8$, (c): LR, $n=8$, (the immature treatment including T5, T6 and T7 was removed from SLR and LR). ** indicate $p<0.01$.
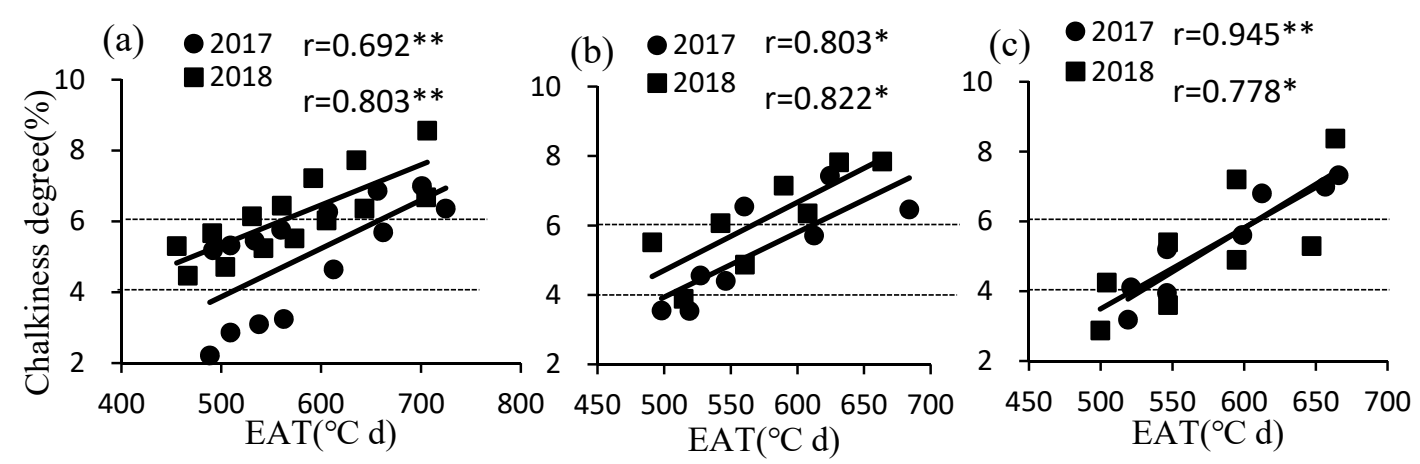

Figure 5. The correlation between chalkiness degree and the EAT of rice at stage of heading-maturity (a): SMR, $n=14$, (b): SLR, $n=8$, (c): LR, $n=8$, (the immature treatment including T5, T6 and T7 was removed from SLR and LR). * and ** indicate $p<0.05$ and $p<0.01$, respectively.
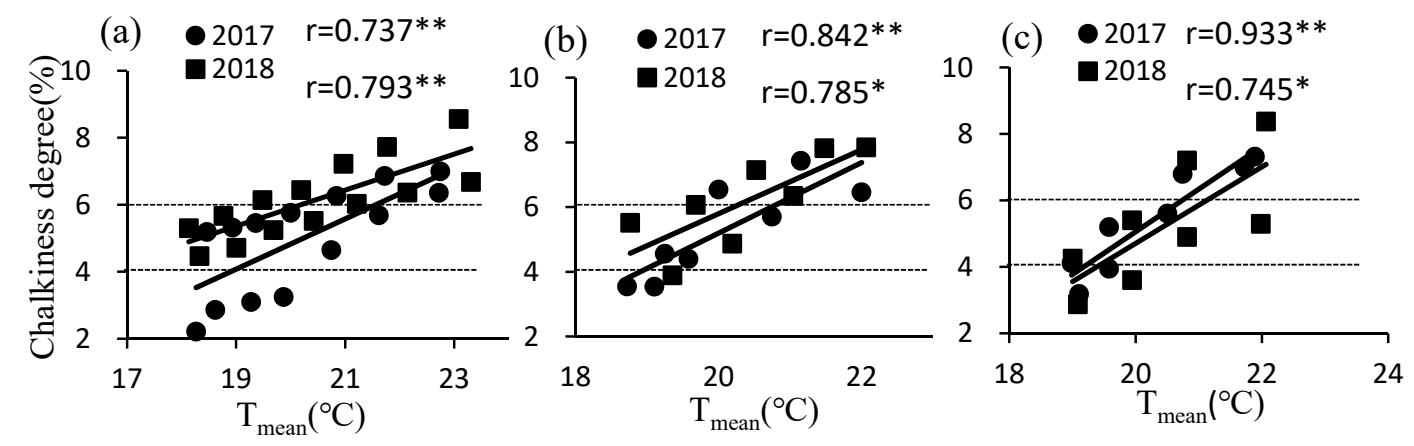

Figure 6. The correlation between chalkiness degree and the $\mathrm{T}_{\text {mean }}$ of rice at stage of heading-maturity (a): $\mathrm{SMR}, n=14$, (b): SLR $n=8$, (c): LR, $n=8$, the immature treatment including T5, T6 and T7 was removed from SLR and LR). * and ** indicate $p<0.05$ and $p<0.01$, respectively. 
Table 6. Characteristics of EAT at stage from heading to maturity of good eating quality rice.

\begin{tabular}{ccccccc}
\hline \multirow{2}{*}{ Type } & \multicolumn{2}{c}{ SMR } & \multicolumn{2}{c}{ SLR } & \multicolumn{2}{c}{ LR } \\
\cline { 2 - 7 } & $\mathbf{2 0 1 7}$ & $\mathbf{2 0 1 8}$ & $\mathbf{2 0 1 7}$ & $\mathbf{2 0 1 8}$ & $\mathbf{2 0 1 7}$ & $\mathbf{2 0 1 8}$ \\
\hline Relatively high-yield & $580.6-724.9^{\circ} \mathrm{C}$ & $574.6-606.3^{\circ} \mathrm{C}$ & $576.1-683.9^{\circ} \mathrm{C}$ & $575.3-663.6^{\circ} \mathrm{C}$ & $576.1-683.9^{\circ} \mathrm{C}$ & $575.3-663.6^{\circ} \mathrm{C}$ \\
GBMI & $450.3-470.4^{\circ} \mathrm{C}$ & $455.4-490.6^{\circ} \mathrm{C}$ & $599.6-683.9^{\circ} \mathrm{C}$ & $623.8-663.6^{\circ} \mathrm{C}$ & $642.0-665.9^{\circ} \mathrm{C}$ & $647.3-663.6^{\circ} \mathrm{C}$ \\
GBMII & $450.3-713.9^{\circ} \mathrm{C}$ & $455.4-708.6^{\circ} \mathrm{C}$ & $431.8-683.9^{\circ} \mathrm{C}$ & $450.7-663.6^{\circ} \mathrm{C}$ & $488.6-665.9^{\circ} \mathrm{C}$ & $400.6-663.6^{\circ} \mathrm{C}$ \\
GBAII & $488.6-508.3^{\circ} \mathrm{C}$ & - & $498.1-502.2^{\circ} \mathrm{C}$ & - & $518.0-528.2^{\circ} \mathrm{C}$ & $499.7-520.7{ }^{\circ} \mathrm{C}$ \\
GBAIII & $488.6-654.3^{\circ} \mathrm{C}$ & $455.4-560.6^{\circ} \mathrm{C}$ & $498.1-609.1^{\circ} \mathrm{C}$ & $491.0-566.2^{\circ} \mathrm{C}$ & $519.0-607.99^{\circ} \mathrm{C}$ & $499.7-606.9{ }^{\circ} \mathrm{C}$ \\
\hline
\end{tabular}

GBMI: The milling quality reaches the first grade of China's national standard of high quality paddy (head rice rate $\geq 67 \%$ ), GBMII: The milling quality reaches second grade of China's national standard of high quality paddy (head rice rate $\geq 61 \%$ ), GBAII The appearance quality reaches second grade of China's national standard of high quality paddy (chalkiness degree $\leq 4.0 \%$ ), GBAIII: The appearance quality reaches third grade of China's national standard of high quality paddy (chalkiness degree $\leq 6.0 \%$ ); "-"indicated that the appearance quality did not reach the second grade of China's national standard of high quality paddy.

Table 7. Characteristics of $\mathrm{T}_{\text {mean }}$ at stage from heading to maturity of good eating quality rice.

\begin{tabular}{ccccccc}
\hline \multirow{2}{*}{ Type } & \multicolumn{2}{c}{ SMR } & \multicolumn{2}{c}{ SLR } & LR \\
\cline { 2 - 7 } & $\mathbf{2 0 1 7}$ & $\mathbf{2 0 1 8}$ & $\mathbf{2 0 1 7}$ & $\mathbf{2 0 1 8}$ & $\mathbf{2 0 1 7}$ & $\mathbf{2 0 1 8}$ \\
\hline Relatively high-yield & $20.2-22.7^{\circ} \mathrm{C}$ & $20.5-23.3^{\circ} \mathrm{C}$ & $20.2-22.0^{\circ} \mathrm{C}$ & $20.4-22.1^{\circ} \mathrm{C}$ & $20.2-22.0^{\circ} \mathrm{C}$ & $20.4-22.1^{\circ} \mathrm{C}$ \\
GBMI & $17.7-18.1^{\circ} \mathrm{C}$ & $18.1-18.8^{\circ} \mathrm{C}$ & $20.6-22.0^{\circ} \mathrm{C}$ & $21.3-22.1^{\circ} \mathrm{C}$ & $21.4-21.9^{\circ} \mathrm{C}$ & $21.8-22.1^{\circ} \mathrm{C}$ \\
GBMII & $17.7-22.7^{\circ} \mathrm{C}$ & $18.1-23.8^{\circ} \mathrm{C}$ & $17.6-22.0^{\circ} \mathrm{C}$ & $18.2-22.1^{\circ} \mathrm{C}$ & $18.4-21.9^{\circ} \mathrm{C}$ & $17.3-22.1^{\circ} \mathrm{C}$ \\
GBAII & $18.3-18.9^{\circ} \mathrm{C}$ & - & $18.7-18.9^{\circ} \mathrm{C}$ & - & $19.0-19.2^{\circ} \mathrm{C}$ & $19.0-19.4{ }^{\circ} \mathrm{C}$ \\
GBAIII & $18.3-21.6^{\circ} \mathrm{C}$ & $18.1-20.2^{\circ} \mathrm{C}$ & $18.7-20.7^{\circ} \mathrm{C}$ & $18.8-20.2^{\circ} \mathrm{C}$ & $19.0-20.7^{\circ} \mathrm{C}$ & $19.0-21.1^{\circ} \mathrm{C}$ \\
\hline
\end{tabular}

GBMI: The milling quality reaches first grade of China's national standard of high quality paddy (head rice rate $\geq 67 \%$ ), GBMII: The milling quality reaches second grade of China's national standard of high quality paddy (head rice rate $\geq 61 \%$ ), GBAII The appearance quality reaches second grade of China's national standard of high quality paddy (chalkiness degree $\leq 4.0 \%$ ), GBAIII: The appearance quality reaches third grade of China's national standard of high quality paddy (chalkiness degree $\leq 6.0 \%$ ); "-" indicated that the appearance quality did not reach the second grade of China's national standard of high quality paddy.

\subsection{Optimum Sowing Date for Good Milling and Appearance Quality Production of Rice}

Under conditions of normal maturity, if the relative grain yield of a given variety is bigger than one, the grain yield of that variety in a certain planting condition is higher than the average yield of that variety in all treatments, indicating a relatively higher grain yield of that variety [19]. The ranges of EAT and $\mathrm{T}_{\text {mean }}$ at the stage from heading to maturity for relative high yields production of SMR, SLR and LR are listed in Tables 5 and 6 [25]. The high temperature at the stage from heading to maturity under early sowing conditions was beneficial to increase yield and milling quality of SLR and LR, while it deteriorated the appearance quality of SMR, SLR and LR. The EAT at the stage from heading to maturity of SMR to obtain high yield and good milling quality are $580.6-713.9^{\circ} \mathrm{C}$ and $574.6-606.3^{\circ} \mathrm{C}$ in 2017 and 2018, and the $\mathrm{T}_{\text {mean }}$ was $20.2-22.7^{\circ} \mathrm{C}$ and $20.5-23.3^{\circ} \mathrm{C}$. The EAT of SLR was $576.1-683.9^{\circ} \mathrm{C}$ and $575.3-663.6^{\circ} \mathrm{C}$, and the $\mathrm{T}_{\text {mean }}$ was $20.2-22.0^{\circ} \mathrm{C}$ and $20.4-22.1^{\circ} \mathrm{C}$, respectively. The EAT of LR to obtain high yield, good milling and appearance quality was $576.1-665.9^{\circ} \mathrm{C}$ and $575.3-663.6^{\circ} \mathrm{C}$, and the $\mathrm{T}_{\text {mean }}$ was $20.2-20.7^{\circ} \mathrm{C}$ and $20.4-22.1^{\circ} \mathrm{C}$, respectively.

EAT is often used to evaluate the accumulation of heat resources of a certain rice variety under certain cultivation conditions [26]. The EAT was taken as the index to use for statistical analysis of the best sowing times in the recent years in this study. It is important to highlight that the daily minimum temperature stably passed $10{ }^{\circ} \mathrm{C}$ was the same as the earliest sowing date for the formation of high yield, good milling, and appearance quality (Table 8). During the years 2007-2016, the date when the daily minimum temperature stably passed $10^{\circ} \mathrm{C}$ in 2011 was significantly later than that in other years, which led to the earliest optimal sowing date in 2011 being more than $7 \mathrm{~d}$ later than that in the other years. The EATs in 2014 and 2015 were $6.04 \%$ and 6.31\% lower than the average EAT in 2007-2018, which resulted in the latest optimal sowing dates in 2014 and 2015 being more than $9 \mathrm{~d}$ earlier than that in the other years [25]. Therefore, compared with the perennial climate, 
the climate in 2011, 2014 and 2015 was abnormal and the optimal sowing date selected by the remaining seven years was representative in the lower reaches of Huai River. The earliest optimal sowing date for three types of rice obtaining high yield and good quality in the lower reaches of the Huai River was 15 May, and the latest optimal sowing dates for SMR, SLR and LR were 1 June, 20 May and 20 May, respectively.

Table 8. The optimal sowing dates for rice to obtain high yield and good quality.

\begin{tabular}{|c|c|c|c|c|c|c|c|}
\hline \multirow{3}{*}{ Year } & \multirow{3}{*}{ EOS } & \multirow{2}{*}{\multicolumn{2}{|c|}{$\begin{array}{l}\text { SMR } \\
\text { LOS }\end{array}$}} & \multirow{2}{*}{\multicolumn{2}{|c|}{$\begin{array}{l}\text { SLR } \\
\text { LOS }\end{array}$}} & \multirow{2}{*}{\multicolumn{2}{|c|}{$\frac{\text { LR }}{\text { LOS }}$}} \\
\hline & & & & & & & \\
\hline & & 2017 & 2018 & 2017 & 2018 & 2017 & 2018 \\
\hline 2007 & $5 / 10$ & $5 / 30$ & $6 / 1$ & $5 / 21$ & $5 / 21$ & $5 / 21$ & $5 / 22$ \\
\hline 2008 & $5 / 15$ & $5 / 31$ & $6 / 2$ & $5 / 21$ & $5 / 22$ & $5 / 21$ & $5 / 22$ \\
\hline 2009 & $4 / 28$ & $6 / 1$ & $6 / 3$ & $5 / 22$ & $5 / 22$ & $5 / 22$ & $5 / 24$ \\
\hline 2010 & $5 / 14$ & $6 / 4$ & $6 / 7$ & $5 / 23$ & $5 / 24$ & $5 / 23$ & $5 / 25$ \\
\hline 2011 & $5 / 23$ & $5 / 26$ & $5 / 28$ & - & - & - & - \\
\hline 2012 & $4 / 18$ & $5 / 30$ & $6 / 1$ & $5 / 18$ & $5 / 21$ & $5 / 18$ & $5 / 21$ \\
\hline 2013 & $4 / 27$ & $6 / 4$ & $6 / 7$ & $5 / 24$ & $5 / 25$ & $5 / 24$ & $5 / 25$ \\
\hline 2014 & $5 / 6$ & $5 / 19$ & $5 / 21$ & $5 / 6$ & $5 / 8$ & $5 / 6$ & $5 / 8$ \\
\hline 2015 & $4 / 23$ & $5 / 18$ & $5 / 22$ & $5 / 6$ & $5 / 6$ & $5 / 5$ & $5 / 6$ \\
\hline 2016 & $4 / 26$ & $6 / 1$ & $6 / 3$ & $5 / 18$ & $5 / 20$ & $5 / 18$ & $5 / 20$ \\
\hline
\end{tabular}

EOS: earliest optimal sowing date; LOS: latest optimal sowing date; " ${ }_{-}$" indicated that there is no suitable sowing date.

\section{Discussion}

\subsection{Response of Milling Quality to Temperature and Solar Radiation}

The filling stage of rice is generally considered to be the key period affecting rice quality $[15,27]$. A low coefficient of correlation was observed between solar radiation and milling quality in this study. Therefore, we believe that the solar radiation resource in this region was abundant, and it was not a limiting factor that affects the formation of good milling quality. The EAT and $\mathrm{T}_{\text {mean }}$ of the three types of rice at the stage from heading to maturity showed a downward trend with the delay of the sowing date. However, the brown rice rate, milled rice rate and head milled rice rate of SMR increased by $0.67-4.09 \%$, $0.80-5.50 \%$ and $0.71-5.23 \%$ respectively. The milling quality of SMR showed a significant negative correlation with EAT and $\mathrm{T}_{\text {mean }}$ at the stage from heading to maturity. Previous studies on the effect of temperature during the rice filling stage on rice milling quality believed that high temperature would increase the amount of broken rice leading to poor milling quality $[15,28,29]$. We supposed that the milling quality of SMR is sensitive to high temperature. It was found that lower temperatures $\left(17.7-18.8^{\circ} \mathrm{C}\right)$ were favorable for SMR forming the first grade of China's national standard of high quality paddy. In contrast, the milling quality of SLR and LR showed significant positive correlation with EAT and $\mathrm{T}_{\text {mean }}$. The temperature requirement of SLR and LR cannot be lower than $20.6^{\circ} \mathrm{C}$ and $21.4^{\circ} \mathrm{C}$ in order to constitute the first grade of China's national standard of high quality paddy. Thus, it appears that the temperature requirements are different for different types of rice, which is consistent with the views of $\mathrm{Li}$ et al. [30]. Increasing temperature in an appropriate range was beneficial to improve the milling quality of the late-maturing rice $[15,31]$. The $\mathrm{T}_{\text {mean }}$ ranges were $20.2-23.3{ }^{\circ} \mathrm{C}(\mathrm{SMR}), 20.2-22.1^{\circ} \mathrm{C}$ (SLR) and $20.2-22.1^{\circ} \mathrm{C}$ (LR), respectively, when the rice obtained relatively high-yields, and the milling quality reached the second grade of China's national standard of high quality paddy. It was considered that late sowing was beneficial for SMR to obtain good milling quality, and early sowing was conducive to improving the milling quality of SLR and LR.

\subsection{Response of Appearance Quality of Good Eating Quality Rice to Temperature and Solar Radiation}

Chalkiness rate and chalkiness degree are the main indexes to evaluate rice appearance quality. The appearance quality of rice is often affected by environmental factors. Many 
studies believe that the high temperature during the grain filling stage will cause the grain refractive index to decrease and formation of chalkiness [32,33]. Compared with solar radiation, a higher correlation coefficient was observed between temperature and chalkiness degree. With the decline in temperature, the chalkiness rate and chalkiness degree of SMR decreased. Interestingly, with the decline in temperature, the chalky grains and chalkiness degree of SLR and LR decreased first and then increased. Gong et al. summarized the effect of low temperature on the appearance and quality of rice and pointed out that the chalkiness is caused by low temperature hindering the division of endosperm cells and reducing the volume of amyloplasts [15]. We believe that low temperature during rice filling stage was beneficial to improve the appearance quality of SLR and LR, but too low temperature is not conducive to the formation of good appearance quality of late-maturing japonica rice.

Under the condition of fully maturity, the appearance quality of the three types of rice failed to reach the first grade of China's national standard of high quality paddy. A very small range of $T_{\text {mean }}$ during the grain filling stage for SMR, SLR and LR to reach the second grade of appearance quality of China National Standard were found, which were 18.3-18.9 ${ }^{\circ} \mathrm{C}, 18.7-18.9{ }^{\circ} \mathrm{C}$ and $19.0-19.4{ }^{\circ} \mathrm{C}$, respectively. The results show that compared with the milling quality, the appearance quality of rice had more stringent requirements in regard to temperature.

Previous research has suggested that a poor transparency of rice with low amylose content was caused by the cavities of starch granules, and the cavity size was negatively correlated with amylose content $[34,35]$. The appearance quality of the three types of rice had a greater range of changes under different sowing dates and the seven sowing dates represented actual field growing conditions. However, under the condition of obtaining a relatively high yield, except for LR, the temperature of SMR and SLR at the stage from heading to maturity cannot meet the appearance quality to reach the third grade of China's national standard of high quality paddy. In addition to the higher $\mathrm{T}_{\text {mean }}$ during grain filling stage, this may also be related to the low amylose content of SMR and SLR [8]. Hence, how to improve the yield and milling quality of SMR and SLR through cultivation measures or variety improvement under the low temperatures needs further research.

\subsection{Recommending an Optimal Sowing Date Range for Good Eating Quality Rice in the Lower Reaches of Huai River}

The rice-wheat rotation is the main planting mode in the lower reaches of Huai River. The annual harvest time for winter wheat in this area is from 1 June to 15 June [36,37]. Considering the time of harvesting, land preparation, and other agricultural consumption, the earliest sowing date and latest maturity date for rice in the lower reaches of Huai River is 16 May and 5 November, respectively. The sowing date ranges for SMR, SLR and LR under the conditions of the rice-wheat double-cropping system were 16 May- 1 June, 16 May-20 May and 16 May-20 May, respectively. The planting area of rice-vegetable, rice-rape and rice-green manure rotations account for $5 \%$ of the double-cropped planting area along the lower reaches of Huai River, and the harvest times are 10-15 days earlier than that of wheat [38]. The optimal sowing dates for the three types of rice were 15 May to 1 June, 15 May to 20 May and 15 May to 20 May, respectively.

\section{Conclusions}

The temperature and solar radiation of six rice varieties showed a decreasing trend at the stage from heading to maturity with the delay of sowing date. Compared with solar radiation, temperature was the main environmental factor affecting the milling and appearance quality of good eating quality rice in the lower reaches of Huai River. Under the condition of obtaining a relatively high yield, the three types of rice can obtain good milling quality. The average temperature and cumulative temperature from May to November in different years have similar changes, although it is difficulties to find every year the same temperature every year during the same sowing days. According to the temperature requirements of different types of rice and the meteorological conditions in the past 10 years, 
we think that the optimal sowing dates for high yield, good milling and appearance quality production of LR was 15 May to 20 May, the optimal sowing dates for high yield, good milling quality production of SMR and SLR were 15 May to 1 June and 15 May to 20 May, respectively. Proposing an appropriate range for the sowing period is beneficial to improve the milling quality and appearance quality of rice in the lower Huaihe River and similar ecological areas. At the same time, finding a suitable growth temperature for rice with good eating-quality is helpful to cope with the decline in yield and quality caused by future warmer climates. However, more experiments are needed, including research on the physiological mechanism of temperature on rice milling and appearance quality.

Author Contributions: Conceptualization, H.W.; methodology, H.Z.; validation, N.Z. and H.W.; formal analysis, N.Z.; investigation, N.Z.; resources, H.W.; data curation, N.Z.; writing-original draft preparation, N.Z.; writing-review and editing, N.Z.; supervision, H.Z.; project administration, H.W.; funding acquisition, H.W. All authors have read and agreed to the published version of the manuscript.

Funding: This research was funded by the National Key Research Program (grant number 2016YFD03 00503); the National Rice Industry Technology System (grant number CARS0127); the National Natural Science Foundation of China (grant number 31971841); the Key Research Program of Jiangsu Province (grant number BE2018355); the Earmarked Fund for Jiangsu Agricultural Industry Technology System, China (grant number JATS[2019]444); and the Project Funded by the Priority Academic Program Development of Jiangsu Higher Education Institutions, China.

Institutional Review Board Statement: The research material is rice and does not require ethical approval

Informed Consent Statement: Not applicable.

Data Availability Statement: Data is contained within the article.

Acknowledgments: We fully appreciate the editors and all anonymous reviewers for their constructive comments on this manuscript.

Conflicts of Interest: The authors declare no conflict of interest. The funders had no role in the design of the study; in the collection, analyses, or interpretation of data; in the writing of the manuscript, or in the decision to publish the results.

\section{References}

1. Du, Y.L.; Zhang, W.J.; Wu, X.R.; Li, G.H.; Wang, S.H.; Liu, Z.H.; Tang, S.; Ding, Y.F. The characteristics of spatial and temporal change of rice yield in Jiangsu Province. J. Nanjing Agric. Univ. 2014, 37, 7-12.

2. Guerrini, L.; Napoli, M.; Mancini, M.; Masella, P.; Cappelli, A.; Parenti, A.; Orlandini, S. Wheat Grain Composition, Dough Rheology and Bread Quality as Affected by Nitrogen and Sulfur Fertilization and Seeding Density. Agronomy 2020, 10, 233. [CrossRef]

3. Cappelli, A.; Oliva, N.; Cini, E. A Systematic Review of Gluten-Free Dough and Bread: Dough Rheology, Bread Characteristics, and Improvement Strategies. Appl. Sci. 2020, 10, 6559. [CrossRef]

4. Zhang, C.X.; Guo, B.W.; Tang, J.; Xu, F.F.; Xu, K.; Hu, Y.J.; Xing, Z.P.; Zhang, H.C.; Dai, Q.G.; Huo, Z.Y.; et al. Combined effects of low temperature and weak light at grain-filling stage on rice grain quality. Acta. Agron. Sin. 2019, 45, 1208-1220.

5. Wu, H.B.; Liu, D.H.; Zhong, M.; Wang, Y.Y. Research progress of climate factor on quality formation and influence mechanism in rice. Hubei Agric. Sci. 2019, 58, 13-18.

6. Cheng, F.M.; Zhong, L.J. Variation of rice quality traits under different climate conditions and its main affected factors. Chin. J. Rice Sci. 2001, 15, 187-191.

7. Cheng, F.M.; Hu, D.W.; Ding, Y.S. Dynamic change of chalkiness and observation of grain endosperm structure with scanning electron microscope under controlled temperature condition. Chin. J. Rice Sci. 2000, 14, 83-87.

8. Ge, G.K.; Zhang, Z.; Shi, C.H.; Wu, J.G.; Ye, Z.H. Impacts of amylose content or protein content on genetic correlations between the weight of brown rice and appearance quality traits of rice. Chin. J. Rice Sci. 2007, 21, 44-50.

9. Cooper, N.T.W.; Siebenmorgen, T.J.; Counce, P.A. Effects of Nighttime Temperature During Kernel Development on Rice Physicochemical Properties. Cereal Chem. J. 2008, 85, 276-282. [CrossRef]

10. Cappelli, A.; Mugnaini, M.; Cini, E. Improving roller milling technology using the break, sizing, and reduction systems for flour differentiation. LWT 2020, 133, 110067. [CrossRef]

11. Cappelli, A.; Oliva, N.; Cini, E. Stone milling versus roller milling: A systematic review of the effects on wheat flour quality, dough rheology, and bread characteristics. Trends Food Sci. Technol. 2020, 97, 147-155. [CrossRef] 
12. Cappelli, A.; Guerrini, L.; Parenti, A.; Palladino, G.; Cini, E. Effects of wheat tempering and stone rotational speed on particle size, dough rheology and bread characteristics for a stone-milled weak flour. J. Cereal Sci. 2020, 91, 102879. [CrossRef]

13. Cappelli, A.; Canessa, J.; Cini, E. Effects of CO2 snow addition during kneading on thermoregulation, dough rheological properties, and bread characteristics: A focus on ancient and modern wheat cultivars. Int. J. Refrig. 2020, 117, 52-60. [CrossRef]

14. Cappelli, A.; Bettaccini, L.; Cini, E. The kneading process: A systematic review of the effects on dough rheology and resulting bread characteristics, including improvement strategies. Trends Food Sci. Technol. 2020, 104, 91-101. [CrossRef]

15. Gong, J.L.; Zhang, H.C.; Hu, Y.J.; Long, H.Y.; Chang, Y.; Wang, Y.; Xing, Z.P.; Huo, Z.Y. Effects of air temperature during rice grain filling period on the formation of rice grain yield and its quality. Chin. J. Ecol. 2013, 32, 482-491.

16. Patindol, J.A.; Siebenmorgen, T.J.; Wang, Y.-J. Impact of environmental factors on rice starch structure: A review. Starch Stärke 2015, 67, 42-54. [CrossRef]

17. Ishimaru, T.; Horigane, A.K.; Ida, M.; Iwasawa, N.; San-Oh, Y.A.; Nakazono, M.; Nishizawa, N.K.; Masumura, T.; Kondo, M.; Yoshida, M. Formation of grain chalkiness and changes in water distribution in developing rice caryopses grown under hightemperature stress. J. Cereal Sci. 2009, 50, 166-174. [CrossRef]

18. Tang, S.; Chen, W.Z.; Liu, W.Z.; Zhou, Q.Y.; Zhang, H.X.; Wang, S.H.; Ding, Y.F. Open-field warming regulates the morphological structure, protein synthesis of grain and affects the appearance quality of rice. J. Cereal Sci. 2008, 84, 20-29. [CrossRef]

19. Xing, Z.-P.; Wu, P.; Zhu, M.; Qian, H.-J.; Hu, Y.-J.; Guo, B.-W.; Wei, H.-Y.; Xu, K.; Huo, Z.-Y.; Dai, Q.-G.; et al. Temperature and solar radiation utilization of rice for yield formation with different mechanized planting methods in the lower reaches of the Yangtze River, China. J. Integr. Agric. 2017, 16, 1923-1935. [CrossRef]

20. Yuan, L.M.; Chang, E.H.; Xu, W.; Wang, Z.Q.; Yang, J.C. Effects of low temperature during grain filling on the structure of endosperm in hybrid rice. Acta. Agron. Sin. 2006, 32, 96-102.

21. Chu, C.Y.; Wang, J.D.; Cheng, Y.; Zhang, Y.; Sun, G.Y. Effect of low temperature treatment in booting and filling stage on yield components and quality of main rice cultivars in Sanjiang Plain. Chin. J. Agrom. 2018, 39, 751-761.

22. Li, T.; Ryu, O.; Tohru, Y.; Haruto, S. Effects of weak light on rice starch accumulation and starch synthesis enzyme activities at grain filling stage. Chin. J. Rice Sci. 2005, 19, 545-550.

23. Ren, W.J.; Yang, W.Y.; Xu, J.W.; Fan, G.Q.; Ma, Z.H. Effect of low light on grains growth and quality in rice. Acta. Agron. Sin. 2003, 29, 785-790.

24. Chen, R.; Ersi, K.; Yang, J.; Lu, S.; Zhao, W. Validation of five global radiation models with measured daily data in China. Energy Convers. Manag. 2004, 45, 1759-1769. [CrossRef]

25. Zhou, N.B.; Zhang, J.; Fang, S.L.; Wei, H.Y.; Zhang, H.C. The effects of temperature and solar radiation on yield in good eating-quality rice grown in the lower reaches of the Huai River Basin. J. Integr. Agric. under review.

26. Wang, W.T.; Zhang, T.F.; Ma, P.L.; Pu, J.Y.; Lü, F.P.; Huang, B. Response of abnormal growth of winter wheat to climate warming in the Loess Plateau of Longdong, Gansu Province. Chin. J. Ecol. 2008, 27, 1492-1497.

27. Song, Z.H.; Zhang, Y.G.; Lu, K.D. Influence of temperature on the super rice polished rice rate and the size of chalkiness in the grouting period. Seed 2009, 28, 66-68.

28. Rehmani, M.I.A.; Wei, G.; Hussain, N.; Ding, C.; Li, G.; Liu, Z.; Wang, S.; Ding, Y. Yield and quality responses of two indica rice hybrids to post-anthesis asymmetric day and night open-field warming in lower reaches of Yangtze River delta. Field Crop. Res. 2014, 156, 231-241. [CrossRef]

29. Zhang, G.F.; Wang, S.H.; You, J.; Wang, Q.S.; Ding, Y.F.; Ji, Z.J. Effect of higher temperature in different filling stages on rice qualities. Acta Agron. Sin. 2006, 32, 283-287.

30. Li, L.; Sha, G.D.; Lu, J.H. Effect of temperature and light factors on rice quality during rice filling stage. Chin. J. Agrom. 1989, 10, 33-38.

31. Dou, Z.; Tang, S.; Chen, W.; Zhang, H.; Li, G.; Liu, Z.; Ding, C.; Chen, L.; Wang, S.; Zhang, H.; et al. Effects of open-field warming during grain-filling stage on grain quality of two japonica rice cultivars in lower reaches of Yangtze River delta. J. Cereal Sci. 2018, 81, 118-126. [CrossRef]

32. Zhang, G.L.; Zhang, S.T.; Wang, L.; Xiao, Y.H.; Tang, W.B.; Chen, G.H.; Chen, L.Y. Effects of high temperature at different times during the heading and filling periods on rice quality. Sci. Agric. Sin. 2013, 46, 2869-2879.

33. Yang, T.T.; Sun, Y.N.; Zeng, Y.H.; Huang, S.; Zhang, J.; Tan, X.M.; Zeng, Y.J.; Pan, X.H. Effect of post-anthesis warming on the grain yield and quality of double-cropped high-quality rice cultivars. J. Nucl. Agric. Sci. 2019, 33, 0583-2591.

34. Zhang, L.; Zhao, L.; Zhang, J.; Cai, X.-L.; Liu, Q.; Wei, C. Relationships between transparency, amylose content, starch cavity, and moisture of brown rice kernels. J. Cereal Sci. 2019, 90, 102854. [CrossRef]

35. Lu, Y.; Zhang, X.M.; Qi, Y.; Zhang, C.Q.; Ling, Y.P.; Liu, Q.Q. Scanning electron microscopic analysis of grain cross-section from rice with different transparency. Chin. J. Rice Sci. 2018, 32, 189-199.

36. Ji, H.J.; Shi, G.Y. Characteristics and High-yielding cultivation techniques of super late sowing wheat in northern Jiangsu Province. Bull Agric. Sci. Technol. 2018, 2, 178-180.

37. Jiang, S.Q.; Xue, D.H.; Jiang, Z.Q. Obstacle factors and countermeasures of high and stable yield of wheat in Yancheng area of northern Jiangsu Province. Mod. Agric. Sci. 2016, 20, 36-38.

38. Shi, G.Y.; Cao, J.H.; Min, H.X.; Gao, D.F. High yield and high efficiency cultivation techniques of rice in greenhouse vegetable stubble. Bull. Agric. Sci. Technol. 2014, 3, 172-174. 